Regular

\title{
Fiber Optic Interrogation Systems for Hypervelocity and Low Velocity Impact Studies
}

\author{
D. A. JACKSON and M. J. COLE \\ Applied Optics Group, School of Physical Sciences, University of Kent, Canterbury, Kent, CT2 7NH, UK. \\ *Corresponding author: D. A. JACKSONＥ-mail: d.a.jackson@kent.ac.uk
}

\begin{abstract}
The aim of this project was to develop non-contact fiber optic based displacement sensors to operate in the harsh environment of a "light gas gun" (LGG), which can "fire" small particles at velocities ranging from $1 \mathrm{~km} / \mathrm{s}-8.4 \mathrm{~km} / \mathrm{s}$. The LGG is used extensively for research in aerospace to analyze the effects of high speed impacts on materials. Ideally the measurement should be made close to the center of the impact to minimize corruption of the data from edge effects and survive the impact. We chose to develop a non-contact "pseudo" confocal intensity sensor, which demonstrated resolution comparable with conventional polyvinylidene fluoride (PVDF) sensors combined with high survivability and low cost. A second sensor was developed based on "fiber Bragg gratings" (FBG) to enable a more detailed analysis of the effects of the impact, although requiring contact with the target the low weight and very small contact area of the FBG had minimal effect on the dynamics of the target. The FBG was mounted either on the surface of the target or tangentially between a fixed location. The output signals from the FBG were interrogated in time by a new method. Measurements were made on carbon fiber composite plates in the LGG and on low velocity impact tests. The particle momentum for the low velocity impact tests was chosen to be similar to that of the particles used in the LGG.
\end{abstract}

Keywords: Intensity sensor, FBG, hypervelocity impacts, composite, high speed processing

\section{Introduction}

The two-stage light gas gun (LGG) is used to study impacts on materials commonly used on space vehicles [1-3] The Kent LGG shown in Fig. 1, fires small projectiles (micron to $\mathrm{mm}$ size) at speeds from $1 \mathrm{~km} / \mathrm{s}$ to $8.4 \mathrm{~km} / \mathrm{s}$. The speed is measured in each shot to be better than $1 \%$ accuracy, and the target chamber is evacuated to typically $0.5 \mathrm{mbar}$ during a shot. Targets can be powered and instrumented during a shot, permitting "real time readout" of any impact sensors on the target. The presence of cosmic dust in space, combined with the growth of man-madedebris in some earth orbits, means that space vehicles are subject to frequent impacts by small high speed objects during their lifetime in space. Equipping space vehicles with impact sensors would help to monitor the growth of this dust flux. Further, real time readout of impact sensors with diagnostic capabilities (e.g. energy or momentum of impact, location of the impact on a larger surface) is potentially a vital tool in monitoring damage to the long term space assets such as the $\$ 100 \mathrm{bn}$ international space station.

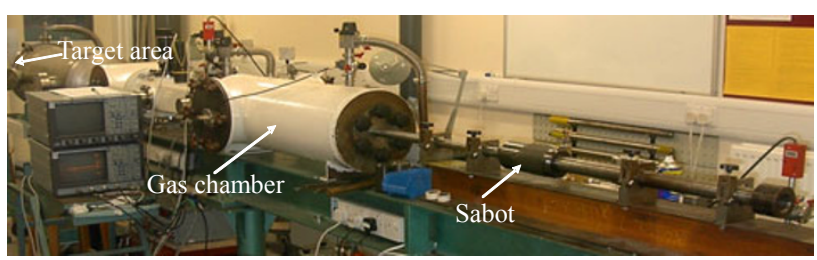

Fig. 1 The light gas gun.

Received: 10 June 2011 / Revised version: 27 June 2011

(C) The Author(s) 2011.This article is published with open access at Springerlink.com 


\section{Intensity sensor}

The intensity sensor based on a confocal configuration [4] is shown schematically in Fig. 2 as it would be aligned with the target. Light from a broad band source at a mean wavelength of $1.50 \mu \mathrm{m}$ is injected into a single mode fiber and transferred by a circulator to an adjustable fiber collimator with a numerical aperture 0.53 and back reflected from $3 \mathrm{M}$ reflective tape mounted on a thin aluminum target. This signal is transferred to a high sensitivity detector and stored in a fast digital "scope", operated in a single shot mode and triggered by a pulse generated by the "particle" passing through a laser gate set across the particle flight path. A signal is also derived from a polyvinylidene fluoride (PVDF) transducer [5] mounted on the side of the target and stored in the 2nd channel of the "scope" such that the PVDF and optical signals are recorded simultaneously after the trigger. Ideally the reflective tape should be placed on the rear of the target directly at the impact site; unfortunately this is not possible as it would be destroyed in the impact, and it was therefore placed on a radius of about $1.5 \mathrm{~cm}$ away from the impact location. Although information is lost, this approach in principle provides more accurate information on the dynamics of the impact than in the case of the PVDF sensor mounted on the edge of the target. To protect the optics a Perspex screen was interposed

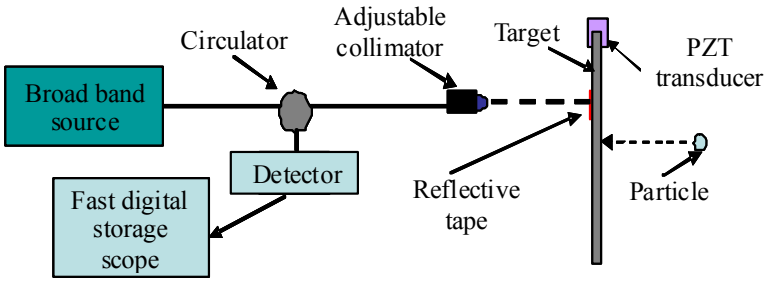

Fig. 2 Optical topology of intensity sensor aligned with the target.

between the collimator and the target. The collimator was mounted about $8 \mathrm{~cm}$ away from the target and tightly focused on the tape to maximize the back reflected optical signal $\left(I_{\max }\right)$. The angular orientation of the collimator was set such that the recovered optical power was $\left(I_{\max }\right) / 2$ prior to the impact to ensure a symmetric intensity signal.

\section{Results obtained with optical sensor for high velocity impacts}

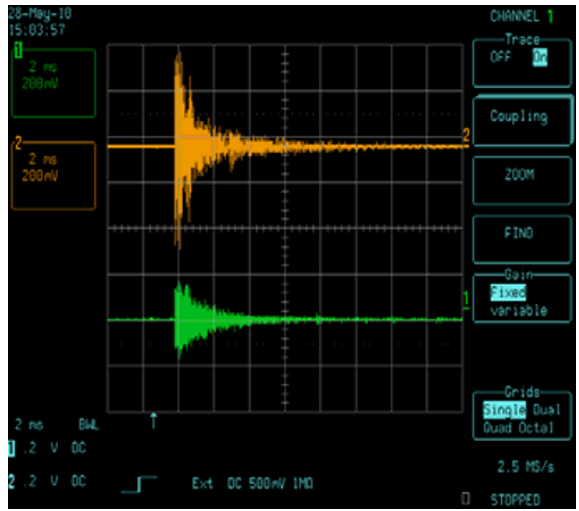

(a) Intensity sensor (top trace) and PVDF sensor (lower trace):

$1.966 \mathrm{~km} / \mathrm{s}$, particle, 1-mm stainless steel ball.

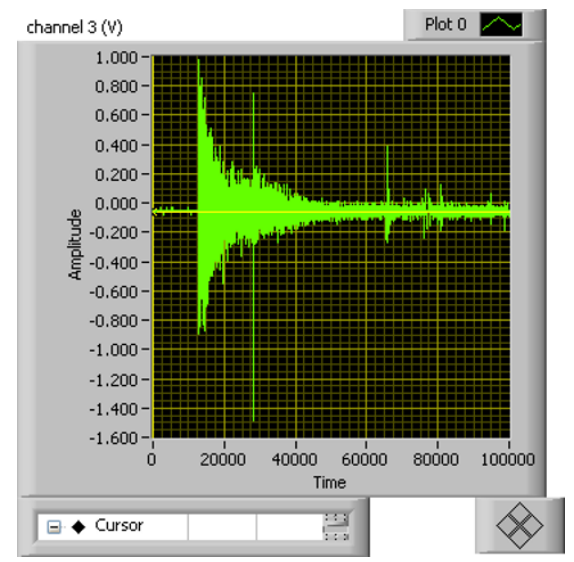

(b) Intensity sensor trace: $3.94 \mathrm{~km} / \mathrm{s}$, particle,

1-mm stainless steel ball.

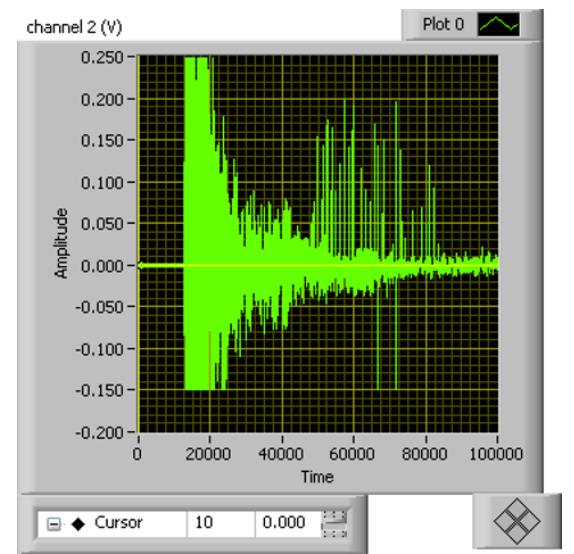

(c) PVDF sensor trace: $3.94 \mathrm{~km} / \mathrm{s}$, particle 1-mm stainless steel ball.

Fig. 3 Output traces generated by a 1-mm ball bearing impacting a thin aluminum target. 
Figure 3(a) shows a direct comparison of the "scope" traces generated by a hypervelocity impact on the target by the edge mounted PVDF contact sensor and the non-contact intensity sensor. It can be seen both sensors have similar response characteristics, which confirm the validity of the optical sensor, and in this example the particle speed was $1.966 \mathrm{~km} / \mathrm{s}$. Figures 3(b) and 3(c) "scope" traces transferred for analysis for a $3.94-\mathrm{km} / \mathrm{s}$ particle. For a shot at $3.94 \mathrm{~km} / \mathrm{s}$, the measured movement of the back of target plate had an estimated maximum speed of $130 \mathrm{~m} / \mathrm{s}$ with peak to peak oscillation amplitude of $260 \mu \mathrm{m}$, for a second shot at $1.069 \mathrm{~km} / \mathrm{s}$ the maximum speed of the back surface was $18 \mathrm{~m} / \mathrm{s}$ with peak to peak amplitude of $42 \mu \mathrm{m}$. With the measured noise of $\pm 9.4 \mathrm{mV}$, around $3 \mu \mathrm{m}$ movements could be seen at the 3 sigma level of detection. The target was a thin aluminum plate.

\section{Interrogation of FBG sensors}

Applications for fiber Bragg gratings (FBGs) [6] have grown very rapidly and have been exploited in many areas including health monitoring of large structures such as bridges [7] and dams [8] , subsea well monitoring [9] and delicate sensors for medical conditions [10]. Many applications require relatively small bandwidths typically less than $1 \mathrm{kHz}$ but relatively high strain resolution of the order of $1 \mu$ strain. Commercial systems are now available from several companies, which fulfill these requirements.

Another rather more specialized area for FBG sensors is that for measuring high frequency perturbations, for example ultrasound [11] and high speed impacts [12]. High frequency signals can be recovered using homodyne or heterodyne [11] techniques. Homodyne signal recovery although offering high resolution[13] can not be readily used to multiplex FBG sensors. Heterodyne signal recovery offers both high frequency signal recovery and the ability to multiplex sensors[14] however the measurement of quasi static signals can be difficult.

Recently there have been several reports of new approaches to recover high frequency large amplitude induced strains in FBGs. Isogo [15] described an interrogation system that used a new form of tunable laser achieving scan rates of $167 \mathrm{kHz}$, with strain rates of less than $500 \mu$ strain at frequencies of $10 \mathrm{kHz}$. Jung [16] described the use of a Fourier domain mode locked laser (FDML) with a maximum scan frequency of $31.3 \mathrm{kHz}$ achieving maximum strain rates of $636 \mu$ strain $(p-p)$ at $100 \mathrm{~Hz}$. Lee [17] also exploited an FDML with a $25.3-\mathrm{kHz}$ scan rate and reported $3000 \mu$ strain at $100 \mathrm{~Hz}$. Lee [18] described a more sophisticated scheme using a linearized FDML achieving $3000 \mu$ strain at $10 \mathrm{kHz}$.

One of the aims of this project was to develop a FBG interrogation system to analyze the effects of high speed impacts on carbon composite plates. The light gas gun described above was used to project small particles (ball bearings) with velocities in the range of $1 \mathrm{~km} / \mathrm{s}-5 \mathrm{~km} / \mathrm{s}$.

Here we report the details of the new method for FBG interrogation, which offers high frequency signal recovery for large amplitude strains with a new approach for displaying the results.

The FBG signals were digitized and recovered in the time domain. The maximum scan rate for the system was equal to or greater than $20 \mathrm{kHz}$ over a $70 \mathrm{~nm}$ range with a demonstrated ability to recover output signals from FBG sensors modulated at frequencies up to $10 \mathrm{kHz}$ and strains up to $5000 \mu$ strain.

The complete experimental integration system is shown in Fig. 4. The output from a broad band Erbium doped super-fluorescent fiber source at a mean wavelength of 1.50 microns is injected into a high finesse fiberized Fabry-Perot (FFP) with a scanning range of about $70 \mathrm{~nm}$. The FFP is driven with a sinusoidal source at frequencies up to $20 \mathrm{kHz}$ via a bias tee. The variable direct voltage applied to the bias tee enables the operating point of the FFP to be adjusted to the linear region of its transfer function. The output signal from the FFP is then injected into the FBG via a circulator. The light back 
reflected from the FBG is transferred by the circulator to a high speed $125 \mathrm{MHz}$ fiber coupled detector.

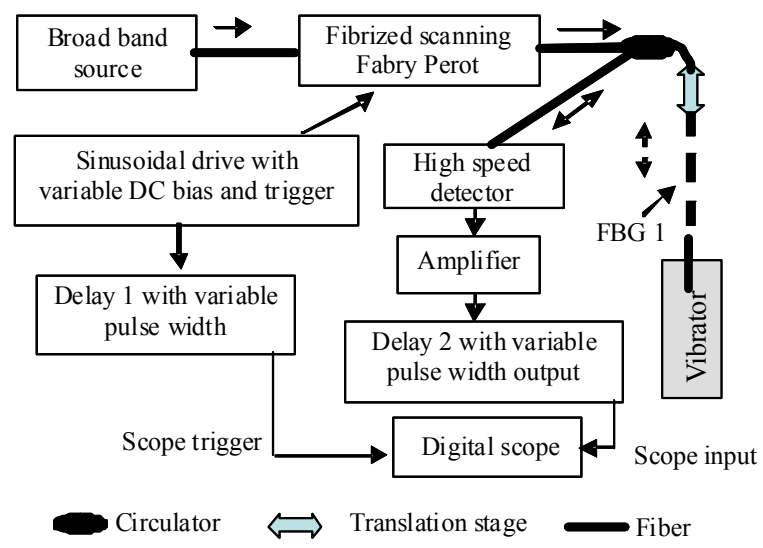

Fig. 4 Interrogation system.

These signals are recovered in the time domain with a very high bandwidth digital scope using a two dimensional waterfall display consisting of a number of segments where the time between segments is equal to the inverse of the system scanning frequency; essentially the sequential " $y$ " axes tick markers in a conventional $x-y$ graph format. The induced changes in the wavelength of the FBG are converted to different times and observed as sequential horizontal scans along the time axis of the waterfall ( $x$ axis), which can be converted to induced strain changes. The FFP sweep is initiated by a trigger pulse that is generated simultaneously with the start of the sinusoidal FFP drive signal. This pulse is transferred to variable delay generator (delay 1) and its output pulse triggers the start of the digital "scope". The output from the detector is amplified and converted into a digital pulse with an edge discriminator (delay 2). The time between the pulse initiating the data collection and the output pulse from delay 2 defines the variation in the amplitude of the applied strain to the FBG on each time segment.

\section{Performance of the FBG interrogator}

In order to establish the ability of the system to analyze the effects of static and frequency dependent strains induced in a FBG the fiber containing the grating was mounted on a translation stage and the other end clamped on the output shaft of a $B \& K$ vibrator, and the separation between mounting points was about $4 \mathrm{~cm}$. The grating could be both dynamically and quasi statically strained at the same time.

\section{Calibration of the time to strain coefficient of the FBG}

Figure 5 shows the calibration curve $\Delta t / \Delta \varepsilon$ where $\Delta t$ is the induced time variation of the output of the FBG for a change $\Delta \varepsilon$ in the applied strain. It was measured in situ with the system scanned at $5 \mathrm{kHz}$. A least squares fit to the graph gave

$$
\Delta t / \Delta \varepsilon=3.52 \pm 0.13 \mu \mathrm{s} / 1000 \mu \text { strain. }
$$

The minimum detectable incremental strain was $10 \mu$ strain (limited by the translation stage available).

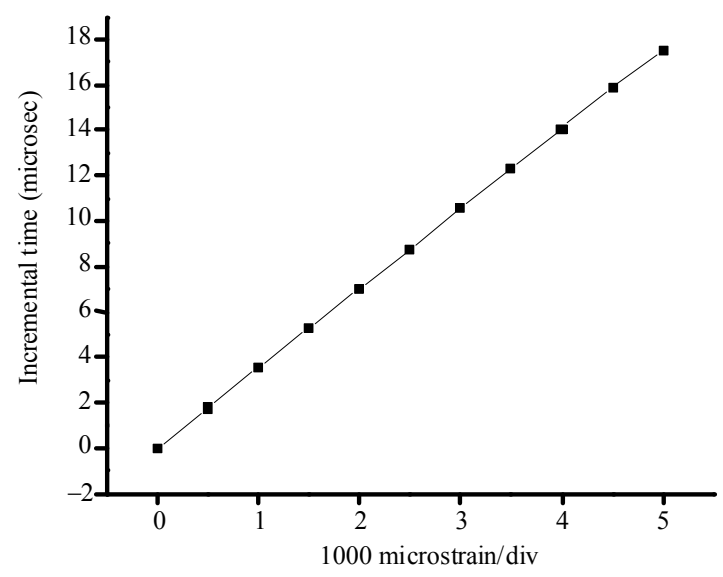

Fig. 5 Plot of the variation of the incremental time in microsecond as a function of applied strain $(1000 \mu$ strain/div $)$.

\section{Test results for the FBG system}

Typical results are shown in Figs. 6 and 7 which were generated when the FBG was subject to various modulation signals. These data were all taken in the linear region of the FP sweep. Figure 6(a) shows the waterfall trace for an FBG modulated at $459.9 \mathrm{~Hz}$, amplitude of about $4000 \mu$ strain, and this data was processed to provide a conventional time verses amplitude plot (Figs. 6(b)) and its fast Fourier 


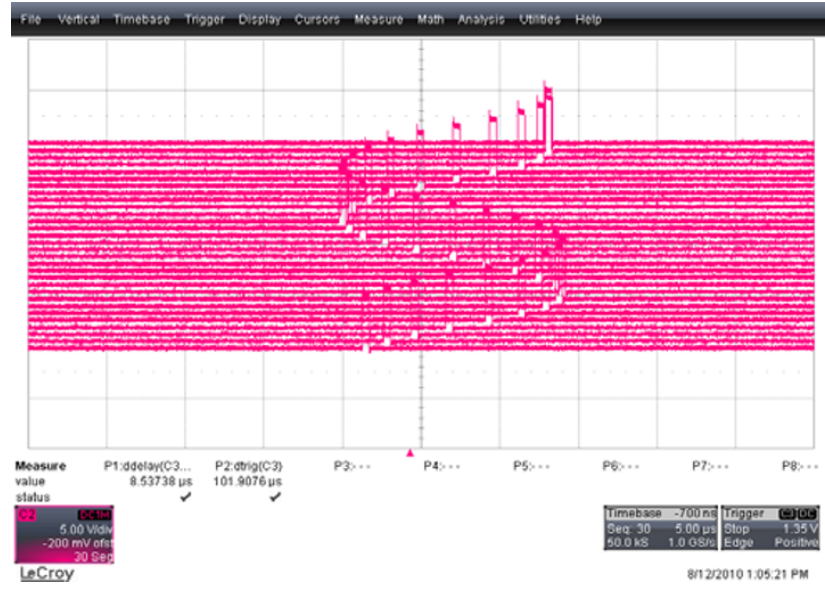

(a) Waterfall plot of the FBG modulated at $495 \mathrm{~Hz}$ at a strain of about $4000 \mu \mathrm{s}$ ( 30 sequences, $5 \mu \mathrm{s} / \mathrm{cm}$ with $1 \mathrm{Gs} / \mathrm{s}$ ).

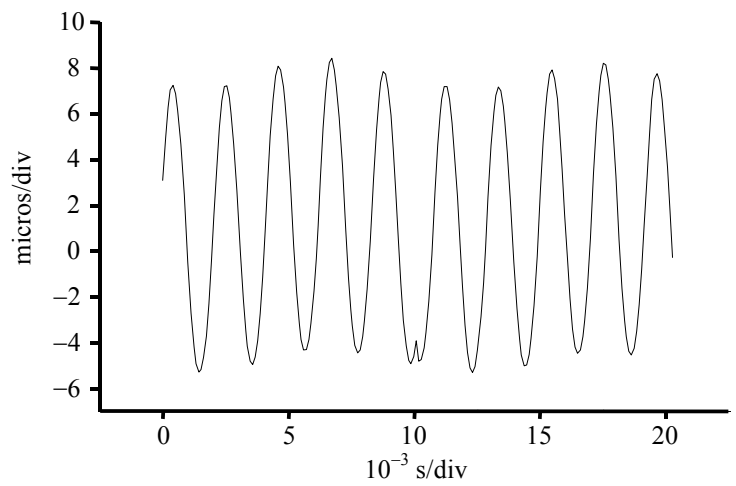

(b) Conventional time verses amplitude plot of data shown in Fig. 6(a).

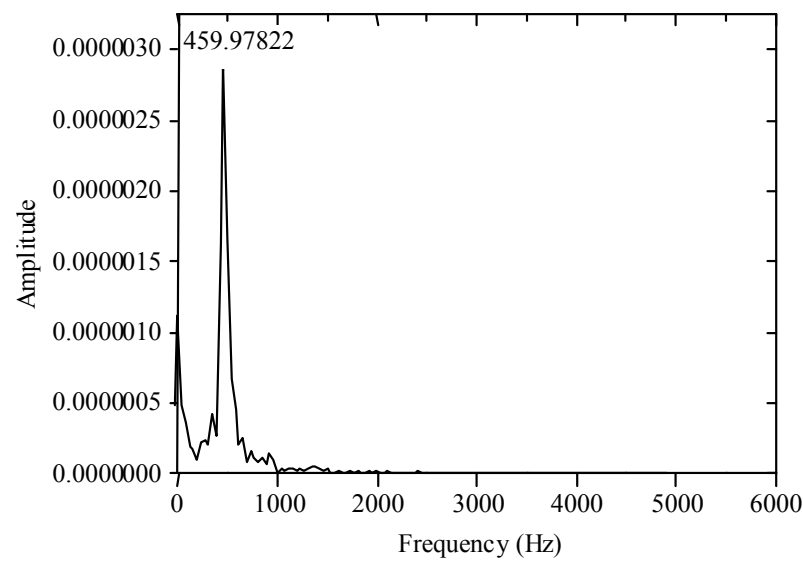

(c) FFT of FBG signal of Fig. 6(b).

Fig. 6 Showing an FBG modulated at $495 \mathrm{~Hz}$ (data is initially recorded as a waterfall plot and subsequently transformed to a conventional plot and its FFT).

transform (FFT) (Fig. 6(c)). Here only 30 sequences were used as it was difficult to see the data on the waterfall plots due to the density of the lines displayed with more sequences, and for most applications sequences of about 1000 would be used in order to achieve a good signal to noise $(\mathrm{S} / \mathrm{N})$. . Figure 7(a) shows the FBG modulated at $5 \mathrm{kHz}$ at strain amplitude of $3130 \mu$ strain; Fig. 7(b) shows a conventional plot of time verses amplitude, and the system scan rate is $20 \mathrm{kHz}$.

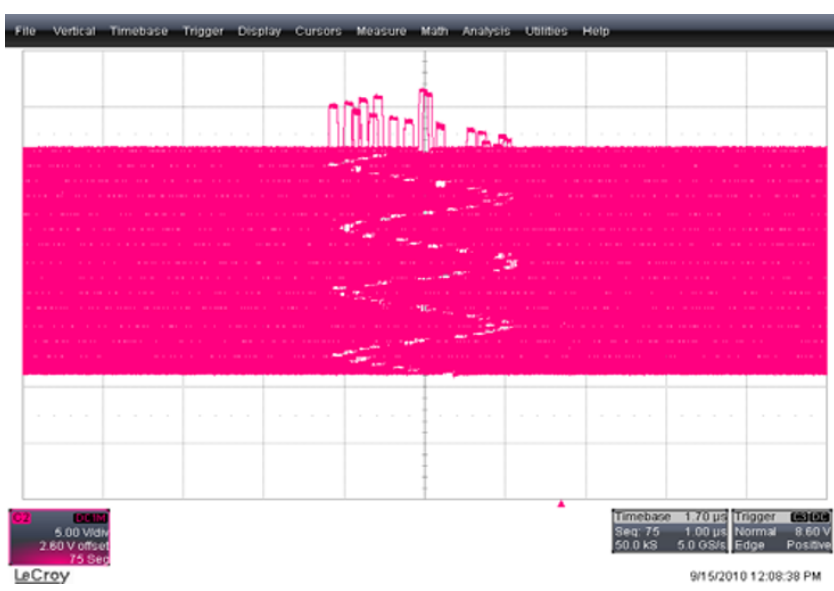

(a) Waterfall plot of FBG modulated at $5 \mathrm{kHz}$ ( 75 sequences, $1 \mu \mathrm{s} / \mathrm{cm}, 5 \mathrm{Gs} / \mathrm{s})$.

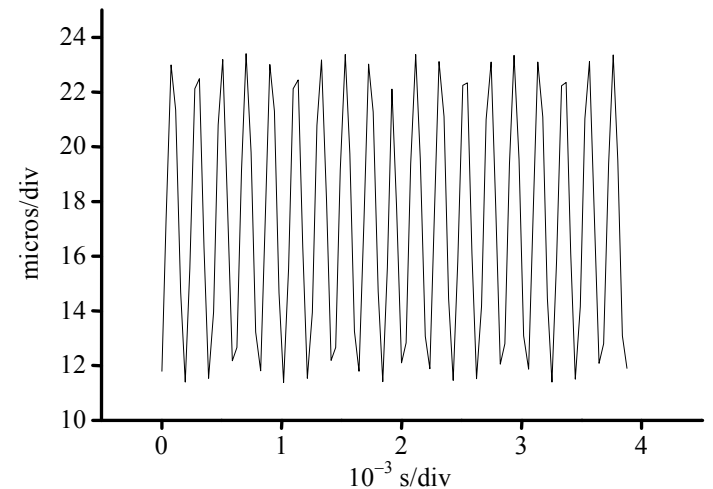

(b) Conventional plot of time verses amplitude of data in Fig. 7(a).

Fig. 7 Showing the waterfall and conventional plots for the same FBG (Fig. 6) modulated at 5kHz.

\section{Mounting the FBG on the target plates}

The targets evaluated for impact studies were square composite plates of $15 \mathrm{~cm} \times 15 \mathrm{~cm}$ and $1.5 \mathrm{~mm}$ thick, with mounting holes at each corner. The plates were mounted on a free standing rigid jig (Fig. 8) designed for the low velocity impact tests or mounting in the LGG. The jig allowed the FBG to be either surface or orthogonally mounted to the plate. 
For surface mounting the FBG was attached at about $1.5 \mathrm{~cm}$ from the center of the plate along a line parallel to the plate edge (SUFBG). In the mounting process the FBG was linearly strained to ensure bidirectional strain response. To enable orthogonal mounting a small hole was drilled through the plate about $2.5 \mathrm{~cm}$ from the plate center. Part of the input fiber just before the FBG was attached to a miniature 3D translation stage. The fiber on the other side of the FBG was passed through the small hole on the surface of the plate and attached to a small fitting. The FBG was then statically strained at about 2 millistrain again ensuring bidirectional strain response (OTFBG).

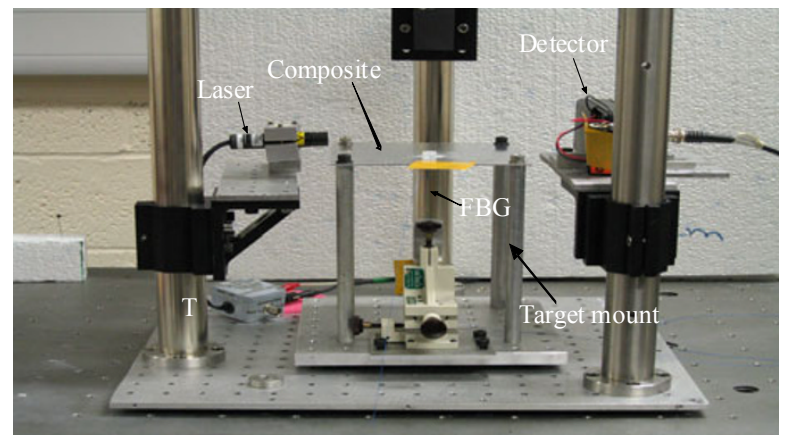

Fig. 8 Arrangement for the low impact test with a laser trigger, which initiates data collection.

\section{Low velocity impact studies}

Prior to making impact measurement at the extreme velocities produced by the LGG, experiments were performed to test that the optical system functioned correctly. This was achieved by studying impacts using much lower impact velocities produced by dropping ball bearings from a small height. The mass and shape of the ball bearings used were the same in both the low velocity and hypervelocity impact studies.

Stainless steel bearings of $8.31 \mathrm{~g}$ or $32.57 \mathrm{~g}$ were used for the low velocity tests. The drop height defined as the distance between the drop-point and the top surface of the plate gave the particle momentum. The heights used were $172 \mathrm{~mm}$ and $169 \mathrm{~mm}$, respectively. The distance between the translator stage attachment points was $6.4 \mathrm{~cm}$.

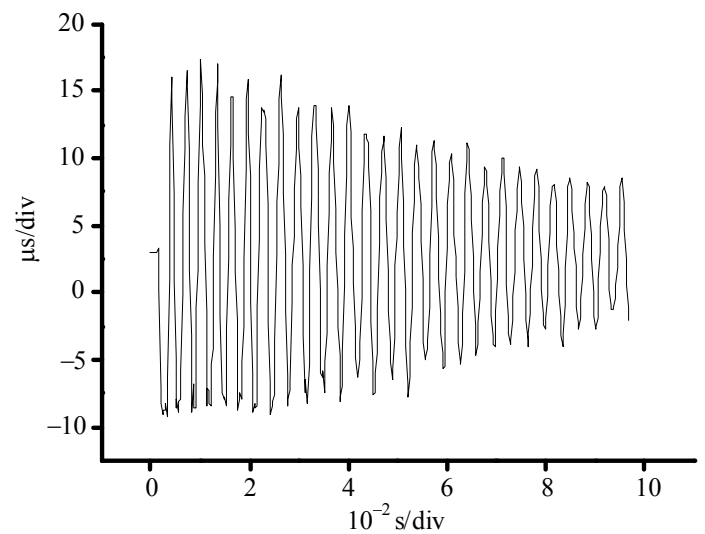

(a) Variation of $\Delta t$ (strain) as a function of time after trigger pulse for OTFBG mounting.

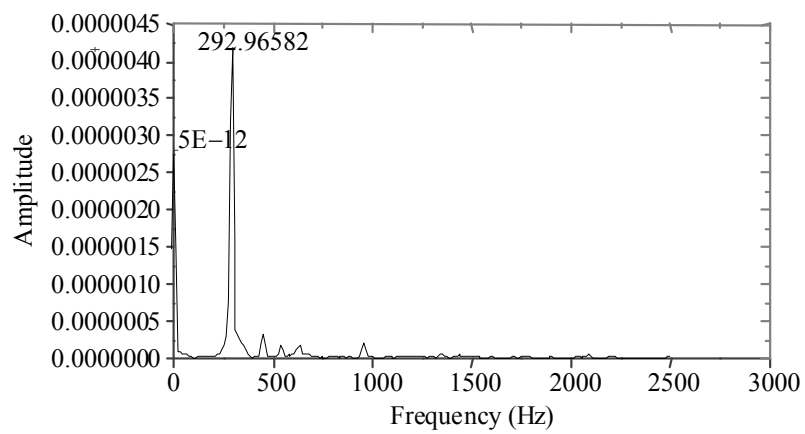

(b) FFT of the data shown in Fig. 9(a).

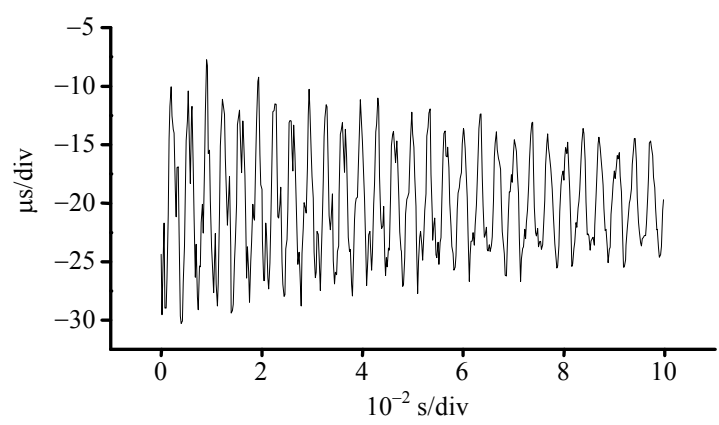

(c) Variation of $\Delta t$ (strain) as a function of time after trigger pulse for SUFBG mounting.

Fig. 9 Results from the low impact studies for FBGs mounted in both configurations.

The stress induced change in the mean reflecting wavelength of the FBG was converted to the time domain as described above. For the measurements reported here the system scanning rate was $12 \mathrm{kHz}$, and the number of sequences in the waterfall was 500 and the time between sequences $200 \mu$ s. In a 
similar study recently reported by [12] the dynamic variation of the spectral profile of the FBG was determined during an impact. Although this was not reported in this paper it would be possible to obtain the same information with the system presented here. To ensure consistent results for the drop tests an optical gate based on a well collimated laser beam was incorporated into the rig. When the ball bearing passed through the beam it opened the gate and initiated data collection.

A series of low velocity impact experiments were performed with the two FBG mounting configurations on composite plates. Figure 9(a) shows the output signal from an OTFBG. Figure 9(b) shows its FFT with a dominate peak at $292.9 \mathrm{~Hz}$. The maximum time deviation of the FBG caused by the impact was converted to strain via (1). The induced strain in the OTFBG of $5680 \mu$ strain was used to determine the "out of plane" displacement of the plate which was $0.436 \mathrm{~mm}$. Figure 9(c) shows the trace for an SUFBG, and the FFT of this trace had a weak peak at $292 \mathrm{~Hz}$ (not shown). There was no evidence of any surface damage to the composite plates.

\section{Experiments performed with the LGG}

A major part of this project was to monitor the effects of hypervelocity impacts on targets mounted in the LGG facility in the space laboratory at the University of Kent. The LGG is used extensively for research in aerospace to analyze the effects of high speed impacts on materials. Ideally the measurement should be made close to the center of the impact to minimize corruption of the data from edge effects and survive the impact. Although the FBG must be mounted in either an OTFBG or an SUFBG configuration, the low weight of the sensors and their very small contact area have minimal effect on the dynamics of the target. Measurements in the LGG were made on similar composite plates to those used in the low speed impact experiments. The particle momentum for the LGG experiments was chosen to be similar to that of the ball bearings used for the low speed impact tests.

\subsection{Hypervelocity impact tests}

Experiments on the LGG were performed on composite plates using the same OTFBG and SUFBG configurations as the low velocity impact tests. Figure 10 shows an OTFBG mounted in the jig (Fig. 8) on the opening door of the LGG, and Fig. 11 shows a normal view of a jig mounted SUFBG also mounted on the LGG door. The traces in Figs. 12(a) and 12(b) are for a composite plate with OTFBG mounting. Figure 12(a) shows the variation of the strain amplitude as a function of time for the FBG signal resulting from a small ball bearing impact at $4.06 \mathrm{~km} / \mathrm{s}$, and Fig. 12(b) is it's FFT which shows a strong peak at $312.5 \mathrm{~Hz}$. The "out of plane" displacement derived from Fig. 12(a) was $0.46 \mathrm{~mm}$. Figure 12(c) shows a small amplitude complex change in the induced FBG strain for SUFBG mounting. The FFT of this signal did not produce any interpretable data.

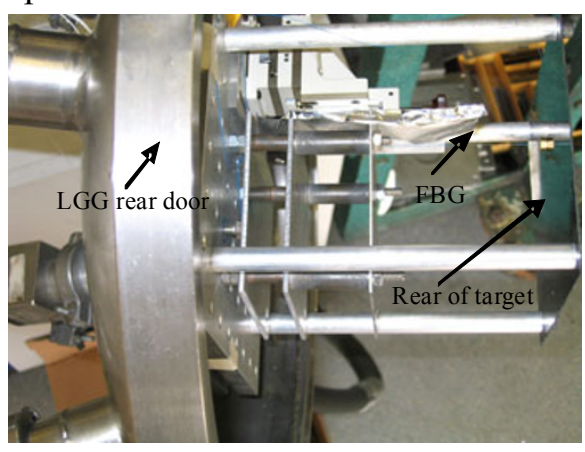

Fig. 10 Target area for an OTFBG mounted sensor.

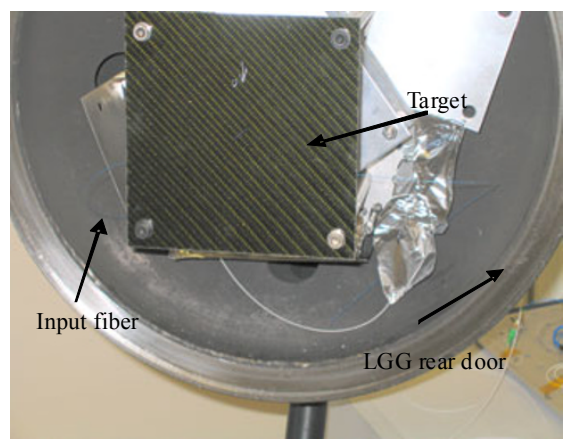

Fig. 11 Target area for an SUFBG mounted sensor. 


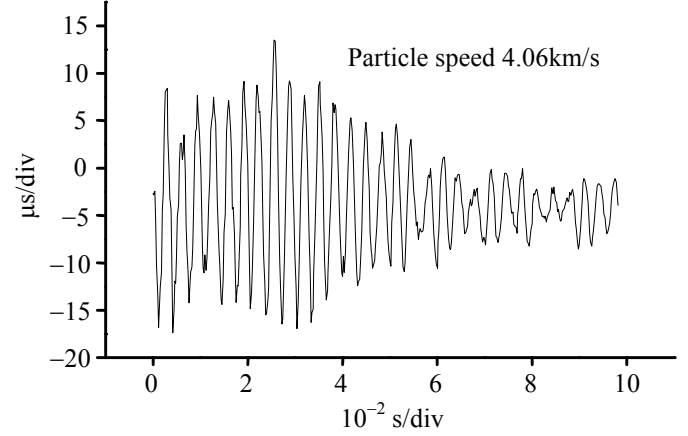

(a) Variation of $\Delta t$ (strain) as a function of time after being impacted at $4.06 \mathrm{~km} / \mathrm{s}$, OTFBG mounted.

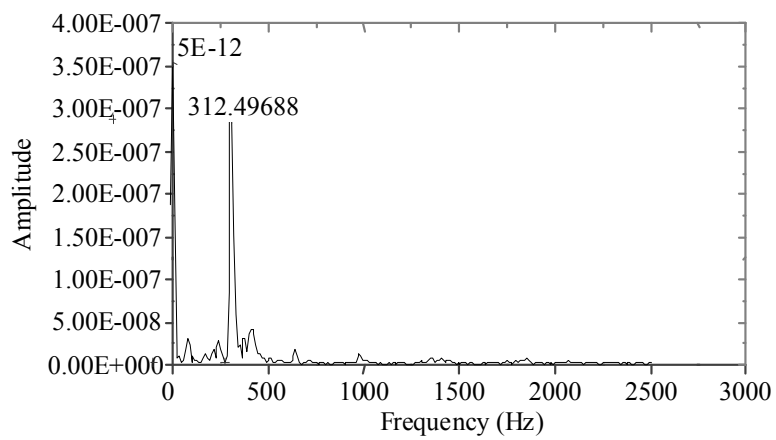

(b) FFT of data from Fig. 10(a).

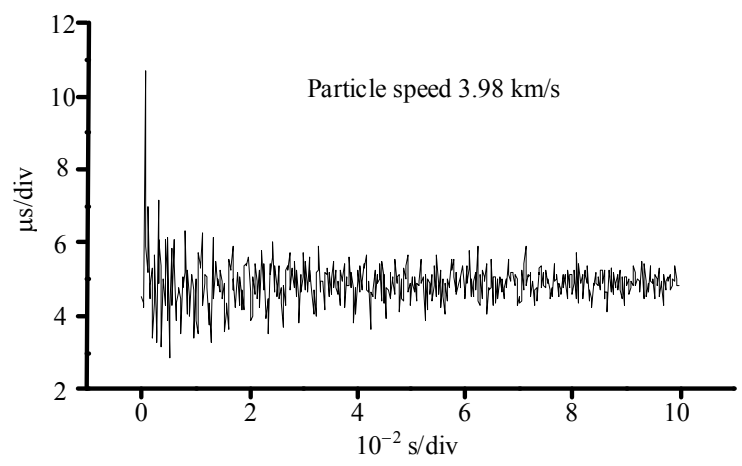

(c) Variation of $\Delta t$ (strain) as a function of time after being impacted at $3.98 \mathrm{~km} / \mathrm{s}$, SUFBG mounted.

Fig. 12 Results for hypersonic impacts on composite plates with OTFG and SUFBG mounted sensors.

In these tests a small hole was punched through the center of the composite plate by the hypervelocity ball bearing causing fine metal dust and carbon (micro) powder to be dispersed in the target-area mitigating against the deployment of conventional optics in the LGG.

\section{Comments}

The intensity sensor target displacement was determined by an ancillary measurement. The target was mounted on a linear scanning translation stage and driven through the intensity profile of the collimator focal profile; the minimum detectable displacement was $3 \mu \mathrm{m}$.

The low velocity impact tests showed that for low momentum impacts high quality signals could be recovered with the OTFBG configuration whereas the result from the SUFBG had a poor S/N The main reason for the poor results for the SUFBG tests is that structural tests would normally be performed with much larger masses [12]. In fact excellent signals could be obtained by simply bending the plates manually.

The signals obtained with the LGG (OTFBG mounting) look promising providing useful results on the targets dynamic motion, whereas the results with SUFBG are not encouraging. The minimum detectable displacement with the OTFBG was $1.24 \mu \mathrm{m}$, and the sensitivity could be improved by using a shorter fiber. The OTFBG mounted on the composite plate gives a minimum momentum detection of $4.4 \times 10^{-5} \mathrm{~N} \cdot \mathrm{s}$, while an estimation for the SUFBG mounting sensitivity is about $1.0 \times 10^{-3} \mathrm{~N} \cdot \mathrm{s}$. Despite the aggressive impacts that the FBGs were subject to, none were broken in either mounting configuration indicating both the strength of the FBGs and the effectiveness of the manner in which they were mounted.

\section{Conclusions}

Two optical fiber based sensors have been developed which produce high quality signals for impact studies. The axial resolution of the confocal intensity sensor was limited by the requirement to operate at a finite distance away from the rear surface of the target. Given the relative simplicity of the optics it would be feasible to deploy several probes in the LGG located in the same plane but at different radii to obtain more information about the dynamics of the target per impact. 
For the low velocity impacts with OTFBG mounting the strain verses time graphs had excellent $\mathrm{S} / \mathrm{N}$ allowing Fourier analysis to be used to determine the vibration spectrum of the composite plate at the time of impact. Although reasonable strain verses time results were obtained with SUFBG mounting the Fourier transform was more complex but had an identifiable main frequency component. These tests were performed with low momentum impacts, and we anticipate that if heavier particles were used as in [12] excellent results would be obtained for both types of FBG mounting.

Experiments with the LGG produced more complicated strain verses time graphs for both FBG mounting methods. Again with the data produced with the OTFBG mounting arrangement a Fourier Transform was used to generate the vibration spectrum of the target, and this could not be achieved for the case of SUFBG mounting. Unfortunately it is not possible with the LGG available at Kent to use larger masses. Given that the time to install a target and pump down the LLG exceeds 5 hours it would be an advantage to exploit the multiplexing [19], capabilities of FBGs in order that more data could be obtained per shot with several sensors mounted strategically on the composite plate, in a serial array.

\section{Acknowledgement}

The authors gratefully acknowledge Prof. Mark Burchell for facilitating access to the LGG. We also acknowledge Dr. Luis Ferreira of Fiber Sensing (Portugal) for supplying the FBGs, Dr. John Nixon of Advanced Composite Group Ltd. (UK) for supplying the composite samples and M. Dimore and P. Henderson from LeCroy Europe for invaluable help in extracting data from the water fall plots.

Open Access This article is distributed under the terms of the Creative Commons Attribution License which permits any use, distribution, and reproduction in any medium, provided the original author(s) and source are credited.

\section{References}

[1] M. J. Burchell, M. J. Cole, J. A. M. McDonnell, and J. C. Zarnecki, "Hypervelocity impact studies using the $2 \mathrm{MV}$ Van de Graaff dust accelerator and two stage light gas gun of the University of Kent at Canterbury," Meas. Sci. Techno., vol. 10, no. 1, pp. 41-50, 1999.

[2] M. J. Burchell, S. Standen, M. J. Cole, R. D. Corsaro, F. Giovane, J-C. Liou, V. Pisacane, and A. Sadilek, "Acoustic response of aluminium and Duroid plates to hypervelocity impacts," Int. Impact Eng,, vol. 38, no. 6, pp. 426-433, 2011.

[3] A. T. Kearsley, M. J. Burchell, M.C. Price, G. A. Graham, P. J. Wozniakiewicz, M. J. Cole, N. J. Foster, and N. Teslich, "Interpretation of Wiid 2 dust fine structure: comparison of Stardust aluminium foil craters to the three-dimensional shape of experimental impacts by artificial aggregate particles and meteorite powders," Meteoritics \& Planetary Science, vol. 44, no. 10, pp. 1489-1509, 2009.

[4] J. H. Bae, K. H. Kim, M. H. Hong, C. H. Gim, and W. Jhe, "High-resolution confocal detection of nanometric displacement by use of a $2 \times 1$ optical fiber coupler," Optics Letters, vol. 25, no. 3, pp. 1696-1698, 2000.

[5] Yi. Meng and Weijian Yi, "Application of a PVDFbased stress gauge in determining dynamic stress-strain curves of concrete under impact testing," Smart Mater. Struct., vol. 20, no. 6, pp. 065004(7 pages),2011.

[6] A. D. Kersey, et al., "Fiber grating sensors," Journal of Lightwave Technology, vol. 15, no. 8, pp. 1442-1463, 1997.

[7] R. L. Idris, K. R. White, J. W. Pate, S. T. Vohra, C. C. Chang, B. A. Danver, and M. A. Davis, "Monitoring and evaluation of an Interstate highway bridge using a network of optical fiber sensors," in Proceedings of the International workshop on Fiber optic Sensors for Construction materials and bridges, Newark, New Jersey, May 3-6, pp. 148-157, 1998.

[8] U. Sennhauser, R. Bronnimann, P. Mauron, and M. Nellen. "Reliability of optical fiber and Bragg grating sensors for Bridge monitoring," in Proceedings of the International workshop on Fiber optic Sensors for Construction materials and bridges, Newark, New Jersey, May 3-6, pp. 117-128, 1998.

[9] A. D. Kersey, "Optical fiber sensor for permanent down-well monitoring applications in the oil and gas industry," IEICE Trans. Electron., vol. E83-C, no. 3, pp. 400-404, 2000. 
[10] Y. J. Rao, D. J. Webb, D. A. Jackson, L. Zhang, and I. Bennion, "Optical in-fiber Bragg grating sensor systems for medical applications," J. Biomed. Opt., vol. 3, no. 1, pp. 38-44, 1998.

[11] N. E. Fisher, D. J. Webb, C. N. Pannell, L. R. Gavrilov, J. W. Hand, L. Zhang, I. Bennion, and D. A. Jackson, "Ultrasonic field and temperature sensor based on short in-fiber Bragg gratings," Electronics Letters, vol. 34, no. 11, pp. 1139-1140, 1998.

[12]A. Propst, K Peters, M.A. Zirkry, W Kunzler, Z. Zhu, N. Wirthlin, R. Selfidge, and S. Schultz, "Dynamic, full-spectral interrogation of fiber Bragg grating sensors for impact testing of composite laminates," in Proc. SPIE, vol. 7503, pp. 75030G, 2009.

[13] B. Culshaw, G. Thursby, D. Betz, and B. Sorazu, "The detection of ultrasound using fiber-optic sensors," IEEE Sensors, vol. 8, no. 7, pp. 1360-1367, 2008.

[14] D. A. Jackson, "Dynamic studies of fiber Bragg gratings," in Proc. SPIE, Fourth European Workshop on Optical fiber Sensors, vol. 7653, pp. 76503, 2010.
[15] R. Isago, K. Nakamura, and S. Ueha, "A high reading rate FBG sensor system using a high-speed swept light source based on fiber vibrations," in Proc. SPIE, vol. 7004, pp. 700411, 2008.

[16] E. J. Jung, C. Kim, M. Y. Jeong, M. K. Kim, M. Y. Jeon, W. Jung, and Z. Chen, "Characterization of FBG sensor interrogation based on a FDML wavelength swept laser," Optics Express, vol. 16, no. 21, pp. 16552-16559, 2007.

[17] B. C. Lee, M. H. Oh, and M. Y. Jeon, "Fiber Bragg grating sensor interrogation with $1.3 \mu \mathrm{m}$ Fourier domain mode-locked wavelength swept laser," in Proc. SPIE, vol. 7503, pp. 75035F, 2009.

[18] H. D. Lee, E.J.Jung, M. Y. Jeong, and C. Kim, "Linearized interrogation of FDML FBG sensor system using PMF Sagnac interferometer," in Proc. SPIE, vol. 7503, pp. 750355, 2009.

[19] Y. J. Rao, A. B. Lobo Ribeiro, D. A. Jackson, L. Zhang, and I. Bennion, "Combined spatial-and time-division-multiplexing scheme for fiber grating sensors with drift-compensated phase-sensitive detection," Optics Letters, vol. 20, no. 20, pp. 2149-2151, 1995. 